\title{
Recomendaciones de cuidados en salud de personas con síndrome de Down: 0 a 18 años
}

División de Pediatría

Escuela de Medicina,

Pontificia Universidad Católica de Chile. aResidente de Cardiología Pediátrica.

Recibido el 25 de noviembre de 2011 aceptado el 25 de abril de 2012.

Correspondencia a: Dra. Macarena Lizama C. Lira $85,5^{\text {to }}$ piso, Santiago, Chile.

Fax: 56-2-6388194

Teléfono: 56-2-3543172/ 56-2-3543753

E-mail:mlizama@med. puc.cl

\author{
MACARENA LIZAMA C., NATALIA RETAMALES M. ${ }^{a}$, CECILIA MELLADO S.
}

\section{Recommendations for health care of people with Down syndrome from 0 to 18 years of age}

People born with Down syndrome have an increased risk of birth defects and are more vulnerable to certain illnesses. Health care for them should emphasize prevention, early diagnosis and early treatment of their most common health conditions. A healthy lifestyle promotes future physical and intellectual development. The purpose of this review is to update health care recommendations for individuals with Down syndrome from 0 to 18 years of age. The health professionals members involved in the care of these patients should be aware of these guidelines.

(Rev Med Chile 2013; 141: 80-89).

Key words: Down syndrome; Primary health care; Trisomy.
$\mathrm{E}$ 1 síndrome de Down (SD) es causado por material extra del cromosoma $21 . \mathrm{Su}$ frecuencia es de 1/700-800 nacidos vivos. En Chile, registros del "Estudio colaborativo latinoamericano de malformaciones congénitas" (ECLAMC) reportan incidencias en ascenso en los últimas décadas, con una tasa de 2,4 por 1.000 nacidos vivos ${ }^{1}$, por otro lado, en países con alto diagnóstico prenatal y leyes de finalización del embarazo, la prevalencia ha disminuido en forma sostenida ${ }^{2-4}$.

La persona con SD se caracteriza por rasgos físicos particulares, mayor riesgo de presentar malformaciones congénitas, mayor vulnerabilidad de enfermedades y discapacidad física e intelectual en grado variable, por lo que requieren de prevención, diagnóstico y tratamiento oportuno de las distintas condiciones de salud que les afectan.

En Chile no existen recomendaciones nacionales respecto a los cuidados en salud de este grupo de personas, por lo que el propósito de esta revisión es actualizar las recomendaciones del cuidado especial desde la etapa prenatal hasta la transición a la vida adulta.

Para esta revisión, se usaron buscadores Pub-
Med, SciELO y revistas de especialidad en SD, en español e inglés.

\section{Cuidados prenatales}

El diagnóstico prenatal es variable, y depende de protocolos locales de pesquisa prenatal y de diagnóstico citogenético disponibles. En Chile, el estudio hormonal materno, que selecciona mujeres con embarazo de riesgo de cromosomopatías, no se realiza de rutina, así, la mayor sospecha diagnóstica se centra en elementos ecográficos. A pesar de que no existen datos estadísticos, esto supone una tasa de sospecha o diagnóstico prenatal más baja que en países en donde la pesquisa es de rutina.

En aquellos con diagnóstico prenatal, el cuidado se centra en la forma de comunicar la noticia a los padres, en la búsqueda de anomalías congénitas asociadas y en la preparación para el cuidado del recién nacido (RN). Cuando un examen de pesquisa resulta sospechoso de $\mathrm{SD}$, se debe plantear a los padres la posibilidad de confirmación diagnóstica, tomando en cuenta los riesgos del procedimiento, 
el beneficio de la confirmación diagnóstica y la oportunidad de preparación de los padres. Los resultados de diagnósticos citogenéticos deben ser entregados por personal entrenado en entregar noticias difíciles y con conocimiento actualizado en SD. La mayoría de las veces, ese experto es el obstetra, quien además es la persona más cercana a los padres en ese momento, aunque puede hacerse acompañar del genetista clínico, neonatólogo o pediatra ${ }^{5}$. La noticia debe ser idealmente entregada con ambos padres presentes, en un ambiente privado, agradable y sin interrupciones, evitando entregar resultados por teléfono o correo electrónico. Se recomienda usar un lenguaje simple y directo, evitando adjetivos negativos (ej: "mongolismo"). Se debe ser honesto, conversar sobre las características positivas, expectativas de vida, enfermedades y discapacidad intelectual (DI). Al finalizar esta visita, es recomendable reforzar la información por escrito ${ }^{6-8}$.

Las visitas sucesivas deben adecuarse a cada mujer embarazada, en ellas se recomienda apoyo de salud mental a los padres, entregar información sobre programas de salud e instituciones que ofrecen apoyo y facilitar contacto con otros padres de personas con $\mathrm{SD}^{6,8,9}$.

La consulta con el genetista es una oportunidad para revisar exámenes diagnósticos, explicar mecanismos patogénicos e informar sobre potencial recurrencia en futuros embarazos y en la familia.

Cuando se han detectado malformaciones congénitas prenatalmente, se recomienda una visita con el neonatólogo para orientar sobre terapias médico-quirúrgicas disponibles y programar el nacimiento en un centro especializado.

\section{Cuidados postnatales}

En Chile, la mayoría recibe el diagnóstico al nacer, cuando las características físicas del $\mathrm{RN}$ hacen sospechar el diagnóstico (Tabla 1) (0-12. $^{12}$

La noticia debe entregarse por el obstetra y/o pediatra, luego que la madre esté recuperada del parto, en un ambiente agradable y privado, junto con el padre y, si se puede, con el RN. Es recomendable comentar la sospecha del diagnóstico por las características físicas encontradas y aclarar que la sospecha será corroborada por un genetista y confirmada con el cariograma9. Es oportuno resaltar las características positivas, las situaciones especiales de salud, en especial las del período neonatal, responder las preguntas de los padres y reforzar lo más importante por escrito ${ }^{8,11}$.

\section{Consideraciones médicas especiales (Guía resumen Tabla 2)}

\section{Atención inmediata}

Verifica la capacidad de adaptación a la vida extrauterina, identifica malformaciones congénitas asociadas, evalúa edad gestacional ${ }^{13} \mathrm{e}$ identifica las características fenotípicas ${ }^{10-12}$ (Tabla 1). En prematuros, pudiera ser difícil identificar con certeza las características habitualmente encontradas en un RN de término con SD, por otra parte, no todos presentan todas las características fenotípicas descritas.

\section{Alimentación y crecimiento}

El adecuado diagnóstico nutricional y de crecimiento se logra con la evaluación del peso, talla y circunferencia craneana en curvas de crecimiento construidas en población con SD. El patrón de crecimiento es bastante similar entre personas con SD, aunque hay diferencias entre países, por lo que algunos han desarrollado curvas propias

Tabla 1. Características físicas del recién nacido con síndrome de Down

Hipotonía
Perfil facial plano
Nariz pequeña y puente nasal bajo
Fisuras palpebrales oblicuas hacia arriba
Manchas de Bruschfield y pliegues epicánticos
Orejas pequeñas, lóbulos auriculares pequeños o ausentes
Boca pequeña y lengua protruyente
Braquicefalia, cuello corto y piel redundante en zona
posterior del cuello
Retraso del cierre de fontanelas
Hiperlaxitud de articulaciones
Pliegue palmar único, hipoplasia de falange media del 5to
dedo y clinodactilia
Espacio entre primer y segundo ortejo ("pie en sandalia")
Diástasis de rectos anteriores
Genitales externos pequeños
Pelo fino, suave y liso


Tabla 2. Recomendaciones del cuidado de salud del niño y adolescente con síndrome de Down

\begin{tabular}{|c|c|c|c|c|c|c|}
\hline & Prenatal & Al nacer & $\begin{array}{c}1-12 \\
\text { meses }\end{array}$ & $\begin{array}{l}1 \text { a } 5 \\
\text { años }\end{array}$ & $\begin{array}{c}5 \text { a } 12 \\
\text { años }\end{array}$ & $\begin{array}{c}12 \text { a } 18 \\
\text { años }\end{array}$ \\
\hline Educación para los padres & $x$ & $x$ & $x$ & $x$ & $x$ & $x$ \\
\hline Cariotipo y consejo genético & $X E$ & $X E$ & & & & \\
\hline Historia y Examen físico & & $x$ & $x$ & $x$ & $x$ & $x$ \\
\hline $\begin{array}{l}\text { Crecimiento y evaluación } \\
\text { nutricional }\end{array}$ & & $x^{*}$ & $x^{*}$ & $x^{*}$ & $x^{*}$ & $x^{*}$ \\
\hline Evaluación del desarrollo & & $x$ & $x$ & $x$ & $x$ & $x$ \\
\hline Educación sexual & & & & & & $X E$ \\
\hline Vacunas $^{\dagger}$ & & $x$ & $x$ & $x$ & $x$ & $x$ \\
\hline \multicolumn{7}{|l|}{ Morbilidad asociada } \\
\hline Cardiología & ECF & EcoCG y E & & & & $E^{\ddagger}$ \\
\hline Gastro-intestinal & ECF & $x^{\S}$ & & ECll & & \\
\hline Evaluación audición & & $T^{t}$ & $E(6 m)$ & E anual & E cada 2 años & E cada 2 años \\
\hline Evaluación visión & & & $E$ & $E$ & E anual & E anual \\
\hline Evaluación dental & & & & $\begin{array}{c}\text { E desde los } 2 \\
\text { años }\end{array}$ & E anual & E anual \\
\hline \multicolumn{7}{|l|}{ Exámenes } \\
\hline Tiroides & & $N^{* *}$ & $N(6$ y $12 \mathrm{~m})$ & $\mathrm{N}$ anual & $\mathrm{N}$ anual & $\mathrm{N}$ anual \\
\hline Hematología & & $\mathrm{H}$ & $\mathrm{H}(12 \mathrm{~m})$ & & & $\begin{array}{l}\mathrm{H} \text { anual en } \\
\text { mujeres }\end{array}$ \\
\hline Rx de col cervical & & & & $\begin{array}{l}3 \text { a } 5 \text { años } \\
\text { (SCC) }\end{array}$ & & \\
\hline PSG o poligrafía & & & & $\begin{array}{l}\text { 3-4 años } \\
\text { (SCC) }\end{array}$ & & \\
\hline
\end{tabular}

X: Evaluar en cada supervisión de salud; ECF: Ecografía fetal; EcoCG: Ecocardiograma; E: Evaluación por especialista; T: Test de Tamizaje (o "screening"); EC: Enfermedad Celíaca; N: nivel hormonas tiroideas (TSH y T4libre); H: hemograma con recuento de

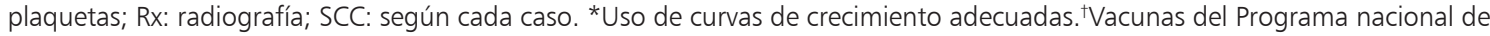
inmunizaciones y vacunas para niños con necesidades especiales. 触 caso de soplo en adolescente sin cardiopatía congénita. EEvaluación clínica de deglución y tránsito intestinal. "Anticuerpos antitransglutaminasa e IgA. "Emisiones otoacústicas. ${ }^{* * T S H, ~}$ T4libre y T3.

como Estados Unidos de Norteamérica ${ }^{14}$, Reino Unido (UK) e Irlanda ${ }^{15}$ y España ${ }^{16}$. La mayoría de ellas no contiene tablas de índice de masa corporal (IMC), parámetro importante para el diagnóstico nutricional de escolares y adolescentes ${ }^{9}$.

Algunos presentan dificultad en ganar peso durante el primer año, principalmente por enfermedades intercurrentes, cardiopatías congénitas (CC) y trastornos de succión-deglución ${ }^{11,17}$.

En escolares y adolescentes el sobrepeso y obesidad ocurren probablemente por recomendaciones nutricionales inadecuadas, sobreprotección de la familia, escasa actividad física y enfermedades no tratadas (ej: hipotiroidismo, depresión).

Recomendaciones: Evaluación de la succión- deglución, fomento de lactancia materna y derivación a fonoaudiólogo para educación de deglución, si hay problemas. Evaluación nutricional con curvas de crecimiento especiales, que en Chile, en ausencia de curvas locales, pudieran usarse las de Pastor ${ }^{16}$ y, en escolares y adolescentes, además evaluar IMC según curvas del National Center of Health Statistics (NCHS) de Estados Unidos de Norteamérica, de acuerdo a la recomendación de la Academia Americana de Pediatría (AAP) ${ }^{9}$.

\section{Neurodesarrollo y problemas neurológicos}

El retraso en la adquisición de hitos del desarrollo y la DI, en la mayoría de los casos es leve a moderada, aunque varía en el tiempo, según 
factores ambientales y de salud.

La estimulación precoz, se conoce como estimulación temprana, tiene por objetivo enseñar habilidades para el aprendizaje y comportamiento y tiene su mayor efectividad cuando se inicia los primeros meses de vida9.

Las convulsiones están descritas hasta en $13 \%{ }^{18}$. El síndrome de West representa hasta $47 \%$ de los síndromes epilépticos en niños con $\mathrm{SD}^{18}$ y donde los espasmos pueden ser confundidos con cólicos del lactante y retrasar el diagnóstico y tratamiento.

Recomendaciones: Derivación precoz a programas de estimulación temprana.

Mantener alta sospecha de episodios sugerentes de crisis convulsivas, estudiar y derivar a neurólogo frente a movimientos anormales, estancamiento del desarrollo o microcefalia. Si bien las convulsiones son más frecuentes, no hay literatura que respalde realizar electroencefalograma de rutina.

\section{Problemas cardiológicos}

Cerca del 50\% presentan algún tipo de $\mathrm{CC}^{9}$, lo que se relaciona a mayor mortalidad y morbilidad en los primeros años de vida ${ }^{19}$. En los pacientes con defectos septales, la cirugía correctora pre$\mathrm{COZ}$ previene el daño vascular pulmonar y sus complicaciones a largo plazo $^{20,21}$. En adolescentes $y$ adultos sin CC, se ha descrito un aumento de valvulopatías ${ }^{22,23}$.

Recomendaciones: Realizar ecocardiografía a todos los RN, aun cuando no existan soplos $\mathrm{u}$ otros síntomas cardiovasculares. En niños sin CC, en caso de aparición de soplos cardiacos, derivar a cardiología para búsqueda de valvulopatías.

\section{Problemas gastrointestinales}

Las malformaciones del tubo digestivo ocurren en $12 \%{ }^{9}$. La sospecha diagnóstica es prenatal o en el período de $\mathrm{RN}$ y en su mayoría son de resolución quirúrgica.

El reflujo gastroesofágico (RGE) y la constipación son frecuentes. El RGE debe ser tratado en forma adecuada para favorecer la calidad de vida y prevenir complicaciones. La constipación ocurre generalmente después de la incorporación de alimentos sólidos y el tratamiento oportuno evita fisuras anales, megacolon o fecaloma. Cuando hay constipación desde el nacimiento o no hay respuesta a terapia médica, se debe sospechar enfermedad de Hirschprung (EH).
La prevalencia de enfermedad celíaca (EC) varía entre 5 y $15 \%{ }^{24,25}$, y hasta un $50 \%$ de los casos pueden presentarse en forma atípica ${ }^{26}$. Aún hay discusión respecto a la utilidad del tamizaje para EC en pacientes asintomáticos.

Recomendaciones: Sospechar malformaciones gastrointestinales según historia clínica y examen físico. No se recomienda ecografía abdominal de rutina en $\mathrm{RN}$ asintomático.

Tratar el RGE y la constipación crónica y mantener alta sospecha de EH.

Para EC, el estudio en asintomáticos es controversial, y en caso de realizarlo, solicitar anticuerpos IgA anti-transglutaminasa e IgA total, luego de haber incluido el gluten en la dieta por al menos 6 meses. Luego de un primer estudio negativo, sólo se recomienda repetir en aquellos con factores de riesgo o sintomáticos ${ }^{9}$ según Tabla 3.

\section{Problemas respiratorios}

Malformaciones de vía aérea y pulmonares (quistes subpleurales, bronquio traqueal y broncomalacia) se han descrito en baja frecuencia ${ }^{27,28}$ y se manifiestan como neumonías recurrentes o atelectasia.

En lactantes con enfermedad pulmonar intersticial y crisis bronquial obstructiva recurrente, se debe sospechar microaspiración.

Las infecciones respiratorias agudas bajas son frecuentes y responsables de la mayoría de la morbilidad no cardiológica que requiere hospitalización. Los lactantes con infección por virus respiratorio sincicial (VRS) tienen riesgo aumentado de hospitalizarse por esta causa, especialmente

Tabla 3. Condiciones de riesgo y síntomas sugerentes de enfermedad celíaca

\section{Condiciones de riesgo}

Antecedentes familiares de Enfermedad Celíaca

Enfermedades autoinmunes relacionadas

Diabetes Mellitus

Hepatitis autoinmune

Tiroiditis autoinmune

\section{Síntomas clínicos sospechosos}

Diarrea

Mal incremento ponderal

Trastorno de conducta

Anemia

Dolor abdominal recurrente

Crecimiento enlentecido 
aquellos con CC, los que además tienen estadías hospitalarias más prolongadas ${ }^{29,30}$. Las sibilancias recurrentes están descritas hasta en 36\% y se asocian con frecuencia a infección previa por VRS o malacia de la vía aérea ${ }^{29}$.

Recomendaciones: Derivación y estudio en niños con historia y síntomas sugerentes de enfermedad pulmonar crónica o en aquellos con sospecha de aspiración. La primera aproximación es con radiografía de tórax, la que no está recomendada como rutina en asintomáticos ${ }^{31}$.

\section{Inmunidad}

Las infecciones respiratorias son frecuentes durante los primeros años, probablemente debido a la hipotonía y anatomía de la vía aérea, más que a un defecto específico del sistema inmune.

Recomendaciones: Optimizar medidas higiénicas, lavado de manos y reducción de contaminación ambiental intradomiciliaria.

En caso de infecciones recurrentes, derivar para estudio de inmunidad.

Fomentar adherencia al programa nacional de inmunizaciones, vacuna anti-influenza y antineumococo. Considerar profilaxis para VRS con anticuerpo monoclonal IgG1 humanizado en grupos de riesgo (Tabla 4$)^{11,32,33}$.

\section{Problemas hematológicos}

La poliglobulia es frecuente e independiente de la presencia de $\mathrm{CC}^{34,35}$. Junto con la trombopenia, son comunes y benignas en las primeras semanas de vida9.

La HCM y VCM pueden estar elevadas, esta "macrocitosis" se observa en cerca de $2 / 3$ de ellos ${ }^{36}$, y hace difícil la orientación diagnóstica en estudios de anemia.

Las reacciones leucemoides son generalmente benignas y de remisión espontánea. En niños

\section{Tabla 4. Grupos de riesgo para considerar profilaxis para VRS}

Cardiopatía congénita cianótica
Insuficiencia cardiaca
Daño pulmonar crónico
Displasia broncopulmonar
Prematuros < 28 semanas, menores de 1 año
Prematuros entre 28 y 32 semanas, menores de 6 meses

mayores y adultos el recuento de leucocitos tiende a ser menor, sin exponerlos a mayor riesgo ${ }^{37,38}$. El trastorno mieloproliferativo transitorio (TMT) es casi exclusivo de personas con SD, ocurre durante las primeras semanas de vida en cerca de $10 \%{ }^{39}$, generalmente es benigna y autolimitada, aunque en algunos puede preceder a leucemia mieloide ${ }^{40,41}$.

La leucemia ocurre en $1 \%{ }^{9}$ y por ello, grupos europeos recomiendan hemograma anual los primeros 5 años $^{10}$, sin embargo, no hay consenso al respecto, por lo que los síntomas clínicos pasan a ser los elementos claves para sospecharla ${ }^{42}$.

El cáncer testicular es más frecuente en comparación con la población general ${ }^{43}$. El tratamiento de la criptorquidia y el examen testicular anual ${ }^{44}$ reduce los riesgos y facilita el diagnóstico precoz.

Recomendaciones: Hemograma con recuento de plaquetas en el RN. Hemograma a los 12 meses para pesquisa de anemia ferropriva y anualmente en mujeres adolescentes que menstrúan ${ }^{9}$. Monitoreo clínico de signos y síntomas sugerentes de leucemia y derivación en citopenias, reacción leucemoide o TMT.

En criptorquidia, derivar a cirujano para orquidopexia antes de los 12 a 18 meses.

\section{Problemas endocrinológicos}

La enfermedad tiroidea ocurre en $28-40 \%$ y aumenta con la edad ${ }^{45,46}$. El hipotiroidismo congénito es 28 veces más frecuente que en la población general $^{45}$. Referencias en Chile muestran hasta $25 \%$ de hipotiroidismo durante el primer año de vida $^{17}$. El hipotiroidismo subclínico es frecuente y reportado como transitorio en $70 \%$ de los $\operatorname{casos}^{45}$, a pesar de ello, aún no hay consenso respecto al reemplazo hormonal en hipotiroidismo subclínico, por lo que el inicio del tratamiento debe decidirse individualmente.

Recomendaciones: TSH y T4 libre a todos los pacientes al nacer, a los 6 y 12 meses y luego anualmente para toda la vida o en caso de síntomas de hipotiroidismo. Derivar a especialista en hipotiroidismo e hipotiroidismo subclínico persistente o sintomático.

\section{Problemas otorrinolaringológicos}

Existe mayor incidencia de estenosis del conducto auditivo externo (CAE), otitis media serosa, infecciones óticas, rinorrea crónica, sinusitis y pérdida de la audición ${ }^{47}$.

El síndrome de apnea obstructiva del sueño 
Recomendaciones de cuidados de salud en personas con Síndrome de Down - M. Lizama et al

(SAOS) es común, incluso con reportes de ocurrencia del $100 \%{ }^{48-51}$, sin embargo, frecuentemente es subestimado por la familia y personal de salud debido a la ausencia de ronquido durante el sueño y a lo inespecífico de las manifestaciones clínicas (Tabla 5) ${ }^{48,51}$.

Recomendaciones: Tamizaje auditivo al nacer: emisiones otoacústicas o potenciales evocados auditivos de tronco. Evaluación clínica de la audición en cada control. Derivación al especialista en: CAE estrecho que impida la visualización, tamizaje alterado o preventivamente a los 6 meses y luego en forma rutinaria toda la vida.

Las recomendaciones internacionales sugieren polisomnograma a los 4 años para evaluación de $\mathrm{SAOS}^{9,51}$. En nuestro medio, el polisomnograma es de alto costo, por lo que sería recomendable estudiar y derivar a aquellos con roncopatía y en aquellos sin ronquido, pero con síntomas asociados (Tabla 5).

\section{Problemas oftalmológicos}

Más de la mitad presentan desórdenes oftalmológicos con necesidad de tratamiento ${ }^{10,52}$. Las cataratas congénitas o adquiridas, obstrucción del conducto nasolacrimal, estrabismo, nistagmus, vicios de refracción, conjuntivitis recurrentes, párpados cortos y oclusión incompleta del ojo, son los problemas más frecuentemente encontrados ${ }^{11,31}$.

Recomendaciones: Rojo pupilar en el control pediátrico. Evaluación por oftalmólogo en los primeros 6 meses y luego anualmente ${ }^{9,52}$. Derivar precozmente en caso de síntomas oftalmológicos según Tabla 6.

\section{Problemas ortopédicos}

La hipotonía e hiperlaxitud aumenta el riesgo de luxación de articulaciones ${ }^{11}$, pies planos y escoliosis. Aproximadamente 15\% presentan laxitud atlantoaxial. Si bien tienen un riesgo aumentado de subluxación atlantoaxial (SAA), sólo 2 a 10\% de ese subgrupo presenta compromiso medular como resultado de accidentes ${ }^{53,54}$. Aún hay discusión respecto al real beneficio de la radiografía cervical como examen de tamizaje, pues no está bien definido que el intervalo atlantoaxial aumentado prediga riesgo de SAA y daño medular, por lo que recientemente la AAP dejó de recomendarla de rutina en asintomáticos?.

Recomendaciones: Radiografía de pelvis a los 3 meses o ecografía antes cuando hay factores de riesgo de displasia de cadera.

Evitar piqueros de cabeza en menores de 6 años ${ }^{9}$, radiografía de columna cervical en niños asintomáticos que se expondrán a actividad física de riesgo. Derivar a traumatología cuando hay síntomas sugerentes de SAA (Tabla 7) ${ }^{53}$, genu valgo, sobrepronación del tobillo, pie plano y escoliosis.

\section{Problemas dermatológicos}

La sequedad de piel y fanéreos es frecuente y facilita grietas, fisuras y sobreinfección. Los problemas que requieren de derivación son: alopecia

\section{Tabla 5. Síntomas inespecíficos en síndrome} de apnea obstructiva del sueño

Despertares nocturnos
Posiciones inhabituales al dormir
Somnolencia durante el día
Falta de energía e iniciativa en actividades escolares
Trastorno del aprendizaje exacerbado
Falta de atención e inquietud psicomotora
Enuresis

Tabla 6. Causas de derivación precoz a oftalmología

Lagrimeo persistente
Conjuntivitis recurrente
Rojo pupilar alterado
Estrabismo
Sensibilidad a la luz
Dolor ocular
Cambios en la visión

Tabla 7. Síntomas sugerentes de Inestabilidad atlanto-axial y compresión medular

Dolor cervical
Cambio en el tránsito intestinal y en la función vesical
Tortícolis
Hiperreflexia
Debilidad muscular
Cuadriparesia o cuadriplejia


areata, vitíligo, eccema seborreico y foliculitis ${ }^{10}$.

Recomendaciones: Educar sobre limpieza, hidratación y lubricación de la piel y uso de protector solar.

\section{Salud mental}

La patología "dual" es hoy día conocida como la concomitancia de donde dos tipos de trastorno mental; en personas con SD, puede existir co-morbilidad entre la DI y los trastornos del comportamiento psiquiatrico, déficit atencional, trastornos emocionales y de ansiedad, autismo, psicosis infantil y trastornos de adaptabilidad social, entre $18 \%$ y $38 \%$ de los niños y adolescentes con $\mathrm{SD}^{55-58}$.

Los factores facilitadores de patologías psiquiátricas se resumen en la Tabla $8^{56,57}$ y es conocido que pueden hacer retroceder las habilidades adquiridas y desencadenar cambios de conducta.

Recomendaciones: Apoyo en salud mental a familia y cuidadores. Controlar factores de riesgo modificables. Derivar a psiquiatra cuando el tras-

\section{Tabla 8. Factores facilitadores y de alerta en patologías psiquiátricas}

Factores facilitadores
Estilos parentales rígidos, ansiosos o muy permisivos
Disfunción familiar severa
Adolescentes con sensación de sentirse inadaptados y
diferentes
Temperamento ansioso y disruptivo
Disfunción práxica y ejecutiva
Déficit de aprendizaje severo
Habla no expresiva, lenguaje repetitivo y pobre compren-
sión del lenguaje
Déficit auditivo y/o visual
Trastornos primarios del sueño
Hipotiroidismo o hipertiroidismo
Hospitalizaciones recurrentes
Elementos de alerta
Inestabilidad emocional
Irritabilidad
Descontrol de impulsos
Autoinjurias
Pérdida de las rutinas de juego
Pérdida de habilidades aprendidas
Alteraciones de la interacción social

torno de la conducta es intenso, cuando resulta en déficit del aprendizaje y socialización o se acompaña de agresividad.

\section{Salud dental}

Los tiempos de dentición temporal y permanente suelen estar atrasados ${ }^{59,60}$. La maloclusión, boca entreabierta, gingivitis y falla en la higiene, desencadena periodontitis, retracción gingival, movilidad dental y caída prematura del diente.

Recomendaciones: Educación sobre aseo dental y evaluación por odontopediatra antes de los dos años y luego anualmente.

\section{Adolescentes}

La edad promedio de la menarquia es 12 años 6 meses y casi la totalidad de las mujeres presentan ciclos ovulatorios regulares y posibilidad de embarazo $^{61,62}$. En los varones, el desarrollo puberal no difiere significativamente con otros adolescentes, sin embargo, las tasas de fertilidad son esporádicas y sólo reducida a reporte de $\operatorname{casos}^{63-65}$. A pesar de lo anterior, se hace imprescindible realizar consejo genético, ya que el riesgo de SD en la generación siguiente es de $50 \%{ }^{64-66}$.

Los jóvenes pueden expresar deseos de tener una pareja y actividad sexual en su vida adulta, sin embargo, los padres y profesionales de la salud son generalmente pesimistas al respecto y muchas veces, se niegan a aceptarlo. En el desarrollo sexual de un adolescente, las barreras psicosociales pueden ser más obstáculo que la limitación propia de su condición ${ }^{61,67}$, por lo que es recomendable derivación a ginecología, educación sexual y elección de métodos de prevención de embarazo adaptados a cada adolescente y adulto, lo que facilitará el desarrollo de una adolescencia saludable, respetando los derechos que toda persona tiene para vivir su sexualidad.

La transición a los cuidados de salud del adulto es compleja. El cambio de cuidadores y la posibilidad de que los proveedores de salud no estén familiarizados con los cuidados especiales, hace que la transición sea dificultosa ${ }^{68}$.

Recomendaciones: Promover cuidados en salud, educación sexual, independencia y autocuidado. Fomentar actividad física, y de esparcimiento y prevenir conductas de riesgo.

Traspasar los cuidados de forma integral al equipo de salud de adultos. 


\section{Conclusiones}

En Chile, el nacimiento de personas con SD es mayor a lo reportado en Latinoamérica, Estados Unidos de Norteamérica y Europa, por lo que ofrecer mejores oportunidades en salud es un desafío. Existen acciones mínimas que están recomendadas para el cuidado en salud de personas con SD, especialmente orientados a detectar en forma precoz malformaciones o enfermedades que interfieren con su desarrollo o que impiden expresar al máximo sus potencialidades.

Las recomendaciones de los cuidados expuestos en esta revisión, son una orientación para los profesionales de la salud, deben ser conocidos por los cuidadores, coordinados por un pediatra, médico de familia o genetista, que eduque a los padres, derive en forma oportuna e integre las recomendaciones de todos los especialistas y facilite la transición a la etapa adulta.

\section{Referencias}

1. Nazer J, Cifuentes L. Estudio epidemiológico global del síndrome de Down. Rev Chil Pediatr 2011; 82 (2): 10512.

2. Roizen NJ, Patterson D. Down's syndrome. Lancet. 2003; 361 (9365): 1281-9.

3. Morris JK, Alberman E. Trends in Down's syndrome live births and antenatal diagnoses in England and Wales from 1989 to 2008: Analysis of data from the National Down Syndrome Cytogenetic Register. BMJ 2009; 339: b3794.

4. Cocchi G, Gualdi S, Bower C. International Trends of Down Syndrome 1993-2004 Birth relation to maternal age and Terminations of pregnancies. Birth Defects Research. 2010; 88: 474-9.

5. Skotko BG, Kishnani PS, Capone GT, Down Syndrome Diagnosis Study Group. Prenatal diagnosis of Down syndrome: how best to deliver the news. Am J Med Genet A 2009; 149A (11): 2361-7.

6. Dent KM, Carey JC. Breaking Difficult News in a Newborn setting: Down Syndrome as a Paradigm. Am J Med Genet C Sem Med Genet 2006; 142C (3): 173-9.

7. Ptacek JT, Eberhardt TL. Breaking bad news. A review of the literature. JAMA 1996; 276 (6): 496-502.

8. Skotko BG, Capone GT, Kishnani PS, Down Syndrome Diagnosis Study Group. Postnatal diagnosis of Down syndrome: Synthesis of the evidence on how best to deliver the news. Pediatrics 2009; 124 (4): e751-8.
9. Bull MJ; Committee on Genetics. Health Supervision for Children With Down Syndrome. Pediatrics 2011; 128 (2): 393-406.

10. Weijerman M, de Winter P. Clinical practice. The care of children with Down syndrome. Eur J Pediatr 2010; 169 (12): 1445-52.

11. Baum RA, Nash PL, Foster JEA, Spader M, RatliffSchaub K, Coury DL. Primary Care of children and adolescents with Down Syndrome: An Update. Curr Probl Pediatr Adolesc Health Care 2008; 38 (8): 241-61.

12. Jones KL. Chromosomal abnormality syndromes: Down Syndrome. En: Smith' Recognizable patterns of human malformation, $6^{\text {th }}$ ed. Elsevier Saunders, 2006, Philadelphia, Pennsylvania-USA. 2006.

13. Tapia JL, Toso P. El recién nacido normal: Atención inmediata, cuidado de transición y puerperio. En: Neonatología. Tapia JL, González A. $3^{\text {ra }}$ ed. Editorial Mediterráneo, Santiago-Chile. 2008.

14. Cronk C, Crocker AC, Pueschel SM, Shea AM, Zackai E, Pickens G, et al. Growth charts for children with Down syndrome: 1 month to 18 years of age. Pediatrics. 1988; 81 (1): 102-10.

15. Styles ME, Cole TJ, Dennis J, Preece MA. New cross sectional stature, weight, and head circumference references for Down's syndrome in the UK and Republic of Ireland. Arch Dis Child 2002; 87 (2): 104-8.

16. Pastor X, Quintó L, Corretger M, Gassió R, Hernández M, Serés A. Tablas de crecimiento actualizadas de los niños con syndrome de Down. Revista internacional sobre Síndrome de Down 2004; 8: 34-46.

17. Retamales N, Moreno R, González A, Cerda J, Lizama M. Down' Syndrome Patient Morbidity and Mortality during the First Year of Life. Rev Chil Pediatr 2009; 80 (4): 323-31.

18. Arya R, Kabra M, Gulati S. Epilepsy in children with Down syndrome. Epileptic Disord 2011; 13 (1): 1-7.

19. Tubman T, Shields M, Craig B, Mulholland H, Nevin N. Congenital heart disease in Down's syndrome: Two year prospective early screening study. BMJ 1991; 302 (6790): 1425-7.

20. Cappelli-Bigazzi M, Santoro G, Battaglia C, Palladino MT, Carrozza M, Russo MG, et al. Endothelial cell function in patients with Down's syndrome. Am J Cardiol 2004; 94 (3): 392-5.

21. Hals J, Hagemo PS, Thaulow E, Sorland SJ. Pulmonary vascular resistance in complete atrioventricular septal defect. A comparison between children with and without Down's syndrome. Acta Paediatrica 1993; 82 (6-7): 595-8.

22. Goldberg-Stern H, Strawsburg RH, Patterson B, Hickey F, Bare M, Gadoth N, et al. Seizure frequency and 
Recomendaciones de cuidados de salud en personas con Síndrome de Down - M. Lizama et al

characteristics in children with Down syndrome. Brain Dev 2001; 23 (6): 375-8.

23. Vis JC, de Bruin-Bon RH, Bouma BJ, Huisman SA, Imschoot L, van den Brink K, et al. Congenital heart defects are under-recognised in adult patients with Down's syndrome. Heart 2010; 96 (18): 1480-4.

24. Bonamico M, Mariani P, Danesi HM, Crisogianni M, Failla P, Gemme G, et al. SIGEP, Medical Genetic Group. Prevalence and clinical picture of celiac disease in Italian Down syndrome patients: A multicenter study. J Pediatr Gatroenterol Nutr 2001; 33 (2): 139-43.

25. Pueschel SM, Romano C, Failla P, Barone C, Pettinato $\mathrm{R}$, Castellano Chiodo A, et al. A prevalence study of celiac disease in persons with Down syndrome residing in the United States of America. Acta Paediatr 1999; 88 (9): 953-6.

26. Rodríguez A, Espín B, González-Meneses A, González M, Pizarro A, Gómez de Terreros I. Down celiac disease profile in Down syndrome patients. Revista médica internacional sobre el síndrome de Down 2010; 14 (1): 3-9.

27. Bertrand P, Navarro, H, Caussade S, Holmgren N, Sánchez I. Airway anomalies in children with Down syndrome: endoscopic findings. Ped Pulm 2003; 36 (2): 137-41.

28. Biko DM, Schwartz M, Anupindi SA, Altes TA. Subpleural lung cysts in Down Syndrome: prevalence and association with coexisting diagnoses. Pediatr Radiol 2008; 38 (3): 280-4.

29. Bloemers BL, van Furth AM, Weijerman ME, Gemke RJ, Broers CJ, van den Ende K, et al. Down syndrome: a novel risk factor for respiratory syncytial virus bronchiolitis: A prospective birth-cohort study. Pediatrics. 2007; 120 (4): e1076-81

30. Hilton JM, Fitzgerald DA, Cooper DM. Respiratory morbidity of hospitalized children with Trisomy 21. Paediatr Child Health 1999; 35 (4): 383-6.

31. Davidson MA. Primary Care for Children and adolescent with Down Syndrome. Padiatr Clin N Am 2008; 55 (5): 1099-111.

32. Corretger JM. Enfermedades susceptibles de vacunación y Síndrome de Down. Revista médica internacional sobre el Síndrome de Down 2008; 12 (2): 18-24.

33. Figueras J, Florez J. Infecciones respiratorias por el virus respiratorio sincicial: prevención en el síndrome de Down. Revista Síndrome de Down 2006; 23: 45-50.

34. Widness J, Pueschel S, Pezzullo J, Clemons G. Elevated erythropoietin levels in cord blood of newborns with Down's syndrome. Biol Neonate 1994; 66 (1): 50-5.

35. Kivivuori S, Rajantie J, Siimes M. Peripheral blood cell counts in infants with Down's syndrome. Clin Genet.
1996; 49 (1): 15-9.

36. Starc TJ. Erythrocyte macrocytosis in infants and children with Down syndrome. J Pediat 1992; 121 (4): 57881.

37. Akin K. Macrocytosis and leukopenia in Down's syndrome. JAMA 1988; 259 (6): 842.

38. Roizen NJ, Amarose A. Hematologic abnormalities in children with Down syndrome. Am J Med Genet 1993; 46 (5): 510-2.

39. Lange B. The management of neoplastic disorders of haematopoiesis in children with Down's syndrome. $\mathrm{Br}$ J Haematol 2000; 110 (3): 512-24.

40. Ma SK, Wan TS, Chan GC, Ha SY, Fung LF, Chan LC. Relationship between transient abnormal myelopoiesis and acute megakaryoblastic leukaemia in Down's syndrome. Br J Haematol 2001; 112 (3): 824-5.

41. Zwaan CM, Reinhardt D, Hitzler J, Vyas P. Acute leukemias in children with Down syndrome. Hematol Oncol Clin North Am 2010; 24 (1): 19-34.

42. Dixon N, Kishnani P, Zimmerman Sh. Clinical Manifestations of Hematologic and Oncologic Disorders in Patients With Down Syndrome. Am J Med Genet C Sem Med Genet 2006; 142C (3): 149-57.

43. Satgé D, Sommelet D, Geneix A, Nish M, Malet P, Vekemans M. A tumor profile in Down Syndrome. Am J Med Genet. 1998; 78 (3): 207-6.

44. Smith DS. Health care management of adults with Down syndrome. Am Fam Physician 2001; 64 (6): 1031-8.

45. Gibson P, Newton R, Selby K, Price D, Leyland K, Addison G. Longitudinal study of thyroid function in Down's syndrome in the first two decades. Arch Dis Child 2005; 90 (6): 574-8.

46. Kishnani P, Crissman B. Current perspectives on Down syndrome: selected medical and social issues. Am J Med Genet C Semin Med Genet 2006; 142C (3): 127-205.

47. Shott SR. Down syndrome: Common otolaryngologic manifestations Am J Med Genet C Semin Med Genet, 2006; 142C (3): 131-40.

48. Marcus C, Keens T, Bautista D, von Pechmann W, Ward S. Obstructive sleep apnea in children with Down syndrome. Pediatrics 1991; 88 (1): 132-9.

49. Levanon A, Tatasiuk A, Tal A. Sleep characteristics in children with Down syndrome. J Pediatr 1999; 134 (6): 755-60.

50. Dyken ME, Lin-Dyken DC, Poulton S, Zimmerman $\mathrm{MB}$, Sedars E. Prospective polysomnographic analysis of obstructive sleep apnea in Down syndrome. Arch Pediatr Adolesc Med 2003; 157 (7): 655-60.

51. Shott SR, Amin R, Chini B, Heubi C, Hotze S, Akers R. Obstructive sleep apnea: Should all children with Down 
syndrome be tested? Arch Otolaryngol Head Neck Surg. 2006; 132 (4): 432-6.

52. Roizen NJ, Mets MB, Blondis TA. Ophthalmic disorders in children with Down. Dev Med Child Neurol 1994; 36 (7): 594-60.

53. Cohen WI. Current dilemmas in Down Syndrome Clinical Care: Celiac Disease, Thyroid disorders, and atlanto-axial instability. Am J Med Genet C Semin Med Genet 2006; 142C (3): 141-8.

54. Pueschel SM, Scola FH. Atlantoaxial Instability in Individuals With Down Syndrome: Epidemiologic, Radiographic, and Clinical Studies. Pediatrics 1987; 80 (4): 555-60.

55. Silka V, Hauser M. Psychiatric Assessment of the Person with Mental Retardation. Psychiatr Ann 1997; 27 (3): 162-9.

56. Summers J, Boyd K, Morgan J. Evaluating patients with intellectual disabilities and comorbid mental health problems. Br J Psychiatry 2004; 34 (3): 215-20.

57. Myers B, Pueschel SM. Psychiatric disorders in persons with Down syndrome. J Ner Ment Dis 1991; 179 (10): 609-13.

58. Gath A, Gumley D. Behaviour problems in retarded children with special reference to Down's syndrome. $\mathrm{Br}$ J Psychiatry 1986; 149: 156-61.

59. Jara L, Ondarza A, Muñoz P, Blanco R. Tiempos de la erupción dentaria temporal en pacientes con síndrome de Down. Rev Chil Pediatr 1995; 66 (4): 186-91.

60. Jara L, Ondarza A, Blanco R. Cronología de la erupción dentaria permanente en pacientes con síndrome de Down. Rev Chil Pediatr 1992; 63 (2): 89-95.

61. Berman H, Harris D, Enright R, Gilpin M, Cathers T, Bukovy G. Sexuality and the adolescent with a physical disability: understandings and misunderstandings. Issues Compr Pediatr Nurs 1999; 22 (4): 183-96.

62. Scola PS, Pueschel SM. Menstrual cycles and basal body temperature curves in women with Down syndrome. Obstet Gynecol 1992; 79 (1): 91-4.

63. Pueschel SM, Orson JM, Boylan JM, Pezzullo JC. Adolescent development in males with Down syndrome. Am JDis Child 1985; 139 (3): 236-8.

64. Sheridan R, Llerena JJ, Matkins S. Fertility in a male with Trisomy 21. J Med Genet 1989; 26 (5): 294-8.

65. Pradhan M, Dalal A, Khan F, Agrawal S. Fertility in men with Down syndrome: a case report. Fertil Steril 2006; 86 (6): 1765.e 1-3.

66. Sutherland GR. Parental autosomal aneuploidy. En: Chromosome abnormalities and genetic counseling. $3^{\text {th }} \mathrm{ed}$. Oxford University Press, New York, NY-USA 2004.

67. Murphy NA, Elias ER. Council on Children With Disabilities. Sexuality of Children and Adolescents With Developmental Disabilities. Pediatrics 2006; 118 (1): 398-403.

68. Lizama M, Avalos ME, Vargas N, Varela M, Navarrete C, Galanti M, et al. Transición al cuidado de la vida adulta, de niños y adolescentes con necesidades especiales de atención en salud: recomendaciones del comité NANEAS de la Sociedad Chilena de Pediatría. Rev Chil Pediatr 2011; 82 (3): 238-44. 